\title{
ESTADO DEL ARTE DE LOS MECANISMOS ALTERNATIVOS DE SOLUCIÓN DE CONFLICTOS EN COLOMBIA*
}

\section{State of the art of alternative mechanisms of conflict resolution in Colombia}

\author{
Rosembert Ariza Santamaría**
}

Fecha de Entrega: 13 de marzo de 2007 Fecha de aprobación: 11 de mayo de 2007 Evaluado por el comité de publicaciones

\section{Resumen}

Esta investigación parte de entender el estado del arte como una investigación documental que tiene un desarrollo propio y cuya finalidad esencial es dar cuenta de construcciones de sentido sobre bases de datos que apoyan un diagnostico y un pronóstico en relación con el material documental sometido a análisis.

Sobre los mecanismos alternativos de resolución de conflictos, diversos autores han realizado estudios desde las siguientes perspectivas: mecanismos alternativos de solución de conflictos como instrumento

Este trabajo forma parte de una investigación en curso que dirige el profesor Ariza Santamaría, con la asistencia de investigación de Gloria Marcela Vera Salazar y con los siguientes estudiantes como auxiliares de investigación: Claudia Nossa, Andrea Chaparro, Lina Aranguren y César Alberto González.

** El profesor Rosembert Ariza Santamaría es docente de la facultad de derecho y director académico del centro de documentación en Mecanismos Alternativos de Solución de Conflictos (MASC). 
para la convivencia, desde el punto de vista procesal se han analizado sus alcances y límites, de igual forma, se han estudiado como mecanismos de acceso a la justicia; y existen algunas posturas sobre los MASC como políticas públicas para, finalmente, revisar los análisis desde una perspectiva cultural.

\title{
Palabras clave
}

Mecanismos alternativos, resolución de conflictos.

\begin{abstract}
This research begins from the study of the state-of-the-art, which is considered like a documentary investigation that has an own development. Its essential purpose is to give account of sense constructions about data bases that support a diagnosis and a foretell regarding the analised documentary material.

Diverse authors have studied the Alternative Mechanisms of Resolution of Conflicts from the following perspectives: The Alternative Mechanisms of Conflict resolution as: an Instrument for the coexistence; its reaches and limits have been analyzed from the procedural point of view, similarly they have been studied as a mechanism of access to justice; and there are some positions about MASC as public policies to finally review the analyses from a cultural perspective.
\end{abstract}

\section{Key words}

Alternative mechanisms, conflict resolution.

\section{INTRODUCCIÓN}

Dentro de los propósitos del Centro de documentación (MASC), de la Facultad de Derecho, está el de promover la investigación y, en este caso, por la especificidad temática, del centro se consideró necesario iniciar por un estado del arte de los MASC en Colombia.

El informe que se presenta a continuación sólo da cuenta de la investigación en curso, que pretende estar terminada en este año. Por ser un informe parcial adolece de varios elementos de rigor académico y, particularmente, de suficiente análisis en algunos acápites. Por ello nada es todavía concluyente.

El estudio parte, entonces, de los trabajos realizados a partir del inicio de la década de los noventa, a partir de la cual se pueden señalar diversos tratamientos sobre el tema de la resolución pacífica de conflictos. Por una parte, se destacan aquellos que han explorado el nivel societal del ejercicio de la justicia, la pertinencia y la contribución de la justicia alternativa a la cultura de la convivencia, y por otra, aquellos que han explorado el aporte de los MASC a la administración de justicia, dentro de los que se destacan, 
el debate respecto al papel de la conciliación extrajudicial, analizando sus aportes y los cuestionamientos que se le plantean particularmente por su impacto como requisito de procedibilidad. Igualmente, estudiaremos aquellos que han ahondado en el aporte de las leyes creadas para instituir los MASC como mecanismos de justicia informal.

A esta lista de perspectivas planteadas, le agregaríamos una bastante actual y desconocida, por algunas instancias. Nos referimos concretamente al enfoque de los MASC y la política pública.

Con estos elementos, y desde un análisis de contexto, se abordará la mirada a la producción y avance teórico en el tema de Justicia informal en CoIombia, empezando, por supuesto, por los Ilamados MASC.

Sin embargo, consideramos que no podemos abordar estos aspectos, sin afrontar el debate aplazado de si los estados del arte pueden o no considerarse investigación.

\section{UNA NECESARIA PRECISIÓN METODOLÓGICA}

Según Graziella Corvalan (1985, P 110), el estado del arte tiene importancia en la medida en que conduzca al conocimiento de los problemas y hallazgos encontrados, así como los vacíos existentes respecto al estudio de un tema determinado. Presenta tres fuentes para su desarrollo:

a. Bibliografía

b. Memoria, es decir, personas que han estudiado el tema.

c. Bases de datos, consistentes en reseñas de publicaciones de un determinado tema.
La noción de estado del arte es relativamente nueva, pues para algunos investigadores se origino en la década de los ochenta 0 , al menos, así ocurrió en Latinoamérica.

Inicialmente, con el estado del arte se pretendía establecer todo lo que se conocía acerca de un tema, buscando como finalidad fundar políticas de acción para el desarrollo de la sociedad.

El autor afirma que el estado del arte convierta a la investigación en un fenómeno de investigación, ya que como fue anunciado anteriormente, el estado del arte es una revisión de lo que sobre un tema se ha producido.

Los presupuestos epistemológicos se derivan de los estudios interpretativos que finalmente $y$ en consecuencia son hermenéuticos. Los estados del arte buscan sintetizar el conocimiento acumulado, dejando al final una larga lista de lo desconocido en determinado tema.

Para conseguir finalmente un conocimiento critico, el estado del arte está compuesto por los siguientes elementos:

1. La recomprensión de fenómenos: se construye cuando se descubren nuevas facetas antes no asociadas con el fenómeno de la investigación, lográndose, gracias a la compilación de evidencias, someterlas a la interpretación, pues en la sistematización deberían quedar plenamente analizadas.

2. La interpretación: que significa que con los estados del arte se busca efectuar una lectura de los resultados alcanzados en los procesos de conocimientos anteriores.

3. La compilación de evidencias: que remite a los procesos alcanzados por la interpretación, ya que solo se puede evidenciar aquello que se conoce. 
4. La reflexión para la acción critica y práctica sobre el conocimiento: el acto de reflexión sobre la teoría es un acto de establecimiento de los límites y posibilidades de una explicación critica sobre la producción teórica.

5. La naturalización de los objetos de estudio: establecimiento del saber que puede ponerse en interrelación con un objeto restringido y deleitado, sobre el que se intenta tanto la aprehensión como la comprensión para su modificación.

En cuanto a los procesos metodológicos, no se conoce de un proceso unificado para realizar un estado del arte, pero se puede decir que generalmente, en éste se define un objeto de estudio, se clasifica la información existente sobre el mismo, se construyen categorías a partir del saber acumulado y se formulan hipótesis sobre las mismas

\subsection{Elementos comunes de los estados del arte}

La contextualización: determinación del problema objeto de estudio.

La clasificación: de la información según parámetros de análisis.

La categorización: las clases permiten a través de descriptores tomar la información que es atinente a lo que se pretende estudiar.

El estado del arte busca identificar lo siguiente (Hoyos, 2000, pp. 55-103):

a. Cuanto se conoce sobre el tema objeto de estudio, conduciendo a la búsqueda de cuanto se desconoce.

b. La ampliación de fronteras.

c. Reinterpretar y actualizar: lo cual quiere decir que se trata de saber cuanto se conoce del tema y si lo que se conoce aun está vigente o cuánto hace falta por conocer de este. Por esta razón, se debe delimitar dentro del estado del arte, el tiempo y el espacio de estudio.

Finalmente, consideramos que el estado del arte es una investigación en todo sentido, pues como lo plantean muchos autores "es una investigación de la investigación". Más allá de ser una simple recolección de datos, posee una ubicación científica, una consideración teórica y un proceso metodológico, que conlleva, al análisis, diagnostico, interpretación y pronóstico de un tema, que permite plantear las hipótesis, se evidencian problemáticas y se dan aportes a posibles soluciones, se observan ventajas, desventajas, eficiencia y eficacia que dejaron investigaciones anteriores a las que se realiza, valorando sus aportes y realizando una sana crítica que permitan generar nuevos caminos de investigación.

\section{MARCO CONSTITUCIONAL Y JURÍDICO DE LOS MASC}

Los mecanismos alternativos de solución de conflictos surgieron a partir de la necesidad latente de la sociedad de solucionar sus conflictos de manera pronta y eficaz, y de la conciencia por parte del Estado de su imposibilidad de solucionar todas las controversias sujetas a su potestad de forma ágil y sin violar garantías fundamentales como la establecida en el artículo 29 de la Constitución Política, "debido proceso". Sobre el surgimiento y necesidad de creación de los MASC, sostienen Mauricio Solórzano y Andrés Felipe Stapper (2004), según su monografía de grado sobre mediación comunitaria:

Un gobierno efectivo, se debe fundamentar en instituciones tanto legales como judiciales prestas a cumplir los objetivos interrelacionados que permitan asegurar el desarrollo del sector priva- 
do, promover el desarrollo del resto de la sociedad y combatir la pobreza. Un sistema judicial que funciona eficazmente es la base del desarroIlo económico... un sistema judicial eficiente está diseñado para resolver conflictos de manera rápida, normatizada e integrada dentro de las costumbres de esa sociedad. Actualmente, los sistemas judiciales latinoamericanos son incapaces de asegurar estas funciones, $y$ de responder a las necesidades, tanto del sector privado como de los habitantes en general, especialmente los más pobres. La reforma de los sistemas judiciales, impulsado parcialmente por instituciones internacionales de desarrollo, es parte de un proceso de cambio global que empieza por redefinir el rol del estado y su relación con la sociedad civil. Hay muchos ejemplos en el continente de los problemas que aquejan hoy a la provisión de justicia. Un listado incompleto de esos problemas incluye, necesariamente, la generalizada falta de independencia del poder judicial... el acceso universal a la justicia y la existencia o no de medios alternativos de resolución de controversias tiene que ser reevaluados. Estos problemas crónicos en los sistemas de justicia latinoamericanos, conjuntamente con regímenes políticos autoritarios, han causado un nocivo impacto psicopolítico en la gran mayoría de la población, manifestada como una generalizada percepción, de la inutilidad o inaccesibilidad de la justicia, la percepción generalizada es que la justicia es demasiado lenta, corrupta, costoso o simplemente ineficiente para resolver problemas de la vida cotidiana de la población.

De acuerdo a lo anterior, se hace palpable que los mecanismos alternativos de solución de conflictos (Masc), nacen además de la falta de prontitud y operancía de la justicia estatal, por la gran desconfianza que generan los órganos estatales encargados de impartir justicia en cada uno de los miembros de la sociedad, por fenómenos tan frecuentes como el de la corrupción, altamente evidenciado en las ramas del poder público.

Por los anteriores motivos, las personas del común prefieren arreglar sus diferencias a través de nuevas formas de justicia, como las pretendidas por Ios MASC.
A partir de la creación de los MASC, nuestro sistema de justicia quedó fraccionado en dos, por un lado la Justicia estatal o formal, la cual, además de pertenecer a las ramas del poder público, se caracteriza por tener una estructura jerárquica no flexible y de dejar al arbitrio de un tercero imparcial de profesión abogado, denominado Juez, la toma de las decisiones dentro del proceso, que se orientan a crear, modificar o extinguir un derecho. No obstante, estar sometidos al impero de la ley, se impone al encargado de impartir justicia la obligación de sustentar sus decisiones en derecho. Por otra parte, encontramos la justicia informal o alternativa, cuya característica principal es la de permitir a las partes el construir la solución a su problema a partir de sus diferentes fórmulas de arreglo con el aval y supervisión de un tercero. Dentro de estos, podríamos nombrar la conciliación, mediación, amigable composición y el arbitraje. No obstante, cabe recalcar que la utilización y viabilidad de estos mecanismos, se sustenta exclusivamente en si el conflicto suscitado versa sobre derechos transigibles, desistibles y conciliables, dado que los conflictos que versan sobre derechos que no se pueden transigir, conciliar, desistir o renunciar, la potestad de decidir sobre estos radica exclusivamente en la Justicia Formal.

El constituyente de 1991 dio a los MASC fundamento constitucional en el inciso 4 del artículo 116 de la Constitución Política, al establecer que los particulares pueden ser investidos transitoriamente de la función de administrar justicia en la condición de conciliadores o en la de arbitros habilitados por las partes para proferir fallos en derecho o en equidad, en los términos que determine la ley. La labor del constituyente de 1991sirvió como preámbulo para empezar a darle legislación debida a los MASC. La primera normatividad de los MASC, la encontramos en la resolución 1116 de 1991, que establecía el trámite y fijaba los requisitos para la organización y funcionamiento de los centros de conciliación, arbitraje y amiga- 
ble composición de las asociaciones, fundaciones, agremiaciones, corporaciones y cámaras de comercio.

\subsection{Marco legal de los MASC}

- La Ley 60 de 1993, artículo 21, numeral 8, dio la facultad a los municipios de coofinanciar el funcionamiento de sus centros de conciliación y comisarías de familia, estas últimas con la labor de hacer las conciliaciones en asuntos de familia.

- La Ley 80 de 1993, artículo 68, faculta a las entidades públicas para utilizar mecanismos de solución directa a las controversias contractuales, para lo cual establece: "Las entidades a las que le son aplicables las normas del estatuto de contratación y así como los contratistas deben buscar en forma ágil, rápida y directa las diferencias y discrepancias surgidas de la actividad contractual".

- Ley 446 de 1998, artículo 113, faculta a las personas jurídicas sin ánimo de lucro para crear centros de arbitraje, previa autorización de la dirección de conciliación y prevención de Ministerio de Justicia y del Derecho. Con esta ley se suprimió la facultad de las entidades públicas para crear centros de conciliación y arbitraje.

- Decreto 1818 del 7 de septiembre de 1998, por el que se crea el estatuto de los Mecanismos Alternativos de Solución de Conflictos, que incorpora en su artículo 226 lo establecido en el artículo 68 de la Ley 80, frente a las controversias contractuales.

- Resolución No. 800 de septiembre 29 del 2000, se establecen los requisitos para la creación de los centros de conciliación y arbitraje, estableciendo así los requisitos que deben cumplir las personas jurídicas sin ánimo de lucro, los consultorios jurídicos de facultades de derecho, de ciencias sociales y humanas $\mathrm{y}$, en general, las personas jurídicas u organismos facultados por la ley, interesados en la creación de centros de conciliación y de arbitraje.

- Ley 640 del 2001, por intermedio de esta se modifican normas relativas a la conciliación y se dictan otras disposiciones, en el artículo 10, se modifica el artículo 66 de la Ley 23 de 1991, al disponer que "las personas jurídicas sin ánimo de lucro y las entidades públicas podrán crear centros de conciliación, previa autorización del Ministerio de Justicia y del Derecho. Los centros de conciliación creados por entidades públicas no podrán conocer de asuntos del contencioso administrativo y sus servicios serán gratuitos". Siendo así se devolvió la facultad a las entidades públicas de contar con dependencias encargadas de prestar servicios de conciliación.

\section{MARCO CONCEPTUAL}

Sobre los Mecanismos Alternativos de Resolución de Conflictos, diversos autores han realizado su estudio desde las siguientes perspectivas: los Mecanismos Alternativos de Solución de Conflictos como:

a) Un Instrumento para la convivencia; b) desde el punto de vista procesal se han analizado sus alcances y límites; c) de igual forma se han estudiado como mecanismos de acceso a la justicia; d)existen algunas posturas sobre los MASC como política pública y finalmente se han realizado análisis desde una perspectiva cultural.

Para Patricia Rodríguez Santana (2001), de acuerdo al informe final a Colciencias sobre las deci- 
siones administrativas y la conciliación como mecanismo alternativo para la solución de conflictos, el conflicto es parte fundamental del cambio social, no obstante es deber de la sociedad y del Estado, convertir los conflictos destructivos, entendidos estos como aquellos en los cuales las partes en litigio resuelven sus diferencias mediante el uso de la violencia, por conflictos constructivos, como aquellos en los cuales las partes optan por la solución pacífica del conflicto a través del consenso. Para lograr esto y por la gran congestión que aqueja la justicia estatal, se hace necesario el recurrir a los MASC. En el caso de la conciliación se debería tomar como parte de la solución, sí se realizan los respectivos estudios de conflictividad en las zonas en las cuales operarán los centros encargados de dicha tarea, para así atender de la manera mas conveniente las necesidades de la sociedad.

Para Mauricio Solórzano y Andrés Felipe Stapper (2004), según su monografía de grado, "Mediación comunitaria", la resolución de conflictos consiste en examinar las dificultades y diferencias de los miembros de la comunidad en disputa, con el propósito de llegar a una solución satisfactoria para las partes y fomentar la integración de la comunidad. En la resolución es importante que todas las partes se sientan involucradas en el proceso y en un plano satisfactorio para cada una de ellas por separado.

La Cartilla Mcanismos alternativos de solución de conflictos, elaborada por el Ministerio de Justicia (1998), está basada en la descripción de los diversos medios generadores de justicia, que permiten a los particulares resolver los conflictos con más persistencia en la sociedad, facilitando a la comunidad, el acceso a procedimientos cortos, sencillos y de bajo costo, promoviendo además un acercamiento de las personas a la auto-solución de sus conflictos y a la ley, fuente y principal origen de los MASC.
Se realiza también un recuento histórico y legislativo de los primeros mecanismos alternativos de solución de conflictos utilizados en el país y cómo han vivido su transformación con el paso del tiempo; todo esto en virtud de la búsqueda de una convivencia pacífica mediante el reconocimiento de las diferencias entre las personas y el incremento de una cultura de paz y equidad conseguida por la sociedad y para la sociedad.

Proceso de seguimiento y evaluación al programa Conciliación en equidad/pedagogía para la paz. BID-Ministerio de Justicia. Elaborado por: Rosa Ludy Arias. 1998

El objeto de la investigación fue realizar una evaluación sobre la formulación y evolución del programa Conciliación en Equidad en el Ministerio de Justicia y del Derecho. El informe presentó una recopilación descriptiva, secuencial y evaluativa de los procesos de formulación, programación y ejecución del programa Pedagogía para la paz/conciliación en equidad.

El estudio está presentado en tres partes: la primera agrupa la evolución de las políticas públicas relacionadas con la resolución alternativa de conflictos, el origen y descripción de la conciliación en Equidad y una propuesta de análisis. La segunda, establece la evolución y el papel desarrollado por el Ministerio de Justicia y del Derecho en la implementación del programa Pedagogía para la paz/conciliación en equidad. La tercera, presenta los logros, limitaciones y el análisis conceptual del programa. Se obtiene también, las conclusiones y recomendaciones sobre el objeto del contrato.

De otra parte, la investigación en la segunda parte, acápite de Memoria Institucional, presenta los cuadros correspondientes al desarrollo institucional de proyecto. Allí se encuentran: etapa inicial (1991-1992), conceptualización y divul- 
gación (1993), institucionalización de los proyectos (1994), ejecución del convenio y evaluación. PNR (1995), inicio de estrategias de descentralización (1996-1997), implementación en cogestión con las universidades (1998). Así también se presenta: un cuadro de cobertura departamental y municipal de la conciliación en equidad (1996): 10 departamentos y 64 municipios y, por último, el cuadro de gestión 1995-1998: Conciliadores en equidad, nombrados (año 1997) 208 y en la casiIla logro 1er. semestre 1998 906, total: 1.114.

Evaluación de la conciliación en equidad, con el propósito de fortalecer el proceso de implementación de la fgura Jueces de paz. Programa de modernización de la administración de Justicia en Colombia USAID-MSD. Elaborado por María Cristina Rodríguez

El documento final de la investigación se encuentra dividido en cuatro partes. La primera llamada marco lógico general, contiene la presentación, los antecedentes de la conciliación en equidad y una parte descriptiva de la metodología. La segunda parte, comprende el procesamiento y análisis de reportes e indicadores. En esta sección se presentan los consolidados generales de reportes cuantitativos y los indicadores de cobertura, acceso, eficiencia y elementos pedagógicos. Se incluyen cuadros y graficas de número de conciliaciones y mediaciones por municipio; participación de conciliadores por departamento; participación de conciliadores por municipio. Un segundo documento integrado dentro del primero presenta: reportes sobre la tendencia de la información, este apartado recoge los resultados generales de los instrumentos aplicados. Igualmente este informe presenta un directorio general de conciliación en equidad, en el consta, entre otros aspectos, el nombre del departamento, los municipios en los cuales hay conciliadores y el número de ellos por municipio; así tenemos un total de 8 departamentos, 70 municipios y 1.181 conciliadores; igual- mente presenta un directorio de conciliadores con los nombres completos, las direcciones, el número de la resolución de nombramiento y la autoridad que la expidió. Es necesario, sin embargo, anotar que el número de conciliadores de este listado no concuerda con el primer directorio mencionado. En el primero, como ya se dijo, se relacionan 1.181 conciliadores, en el segundo, se registran apenas 667 .

La Investigación Que los relatos cuenten nuestras experiencias", elaborada por la Cámara de Comercio y la Alcaldía Mayor de Bogota (2005), está basada en los aportes de los actores voluntarios de convivencia, mediadores comunitarios, conciliadores en equidad y jueces de paz de la ciudad de Bogotá, porque tiene como finalidad la búsqueda de nuevas acciones para la resolución de conflictos y el aumento del acceso a la justicia comunitaria, mediante la sistematización como un proceso de reconstrucción y reflexión de la convivencia comunitaria, teniendo como objetivo principal la consolidación de los procedimientos alternativos de la justicia.

Rosembert Ariza Santamaría (2006), en la cartilla Conciliación en equidad y justicia formal, elaborada para jueces formales, toma como contenido central el de dar a conocer la importancia de esta herramienta en la solución de conflictos en la Administración de justicia, con el fin de lograr un mayor entendimiento y acercamiento entre ambas justicias, que están destinadas a trabajar en forma articulada, para generar una dinámica que garantice un acceso democrático a la justicia.

Alba Lucía Sequeda Gamboa (1999) realizó la investigación "justicia comunitaria y conciliación en equidad, sistematización de la experiencia en Santander", hace un recorrido histórico de antecedentes y origen de la justicia comunitaria en América Latina, y sobre el fortalecimiento de formas alternativas de resolver conflictos en las co- 
munidades, teniendo como fin la construcción de una sociedad colombiana más participativa y justa. La autora plantea en la investigación, el papel que los conciliadores deben tener para que de esta forma la justicia alternativa se pueda evitar toda clase de reducción que castre la potencialidad y perspectiva de los mecanismos de los MASC.

El documento escrito por Miguel Álvarez, Lino Corzo Y David Figueroa (2005), "Jueces de Paz, una figura de la justicia comunitaria: análisis", tiene como objetivo mostrar un diagnostico y hacer un razonamiento sobre los jueces de paz tanto de su surgimiento, proceso de aprendizaje, práctica y como apoyo para el aparato jurisdiccional ordinario, a su vez, como elemento integral de la solución alternativa de conflictos de carácter extrajudicial. Este análisis parte de la observación de diferentes experiencias en el ejercicio de este tipo de justicia, visto como fenómeno relativamente nuevo en el plano práctico en Colombia, aunque, cuente con un respaldo tradicional, constitucional y legal, antiguo.

Este diagnóstico, lejos de ser concluyente, es a ser reflexivo y propositivo, al plantear la justicia de paz como un modelo que tiene en principio, por esencia, da prelación en su aplicación a la conciliación y la mediación. Al nacer el conflicto y la solución en la comunidad, orientada bajo sus lineamientos, por miembros de la misma. Sin embargo, presenta a la vez, falencias de tipo estructural, político, institucional, y personal, ante fenómenos como son la falta de apoyo del Estado ante la carencia de recursos económicos y materiales, la ineficiencia en la aplicabilidad y el cumplimiento de decisiones decretadas bajo la justicia comunitaria y en equidad, la insuficiente divulgación de la figura, y el requerimiento de lineamientos claros y sostenibles. Martha Cecilia Abella y Alfredo Vargas Ortiz (2000), en su texto Justicia de paz y solución de conflictos cotidianos en Neiva, Ibagué y Florencia, reflejan el desarrollo de la investigación respecto al sistema judicial y de desjudicialización, y la participación de la comunidad en la resolución de conflictos, mediante la conciliación en equidad y la justicia de paz. Se abordan estos lineamientos desde su significado, antecedentes, elementos y manejo aplicados en la región sur colombiana, exponiendo el caso de su implementación y, en ella, la variante según el tipo de población (urbana o rural) y la cultura como elemento generador de relaciones sociales que constituyen el conflicto y la forma de solución del mismo, donde factores, como la miseria y la ignorancia, pueden dar giro a una aplicabilidad inadecuada de la justicia de paz por una posible capacitación pobre o nula. Frente a ello surge la necesidad de acompañamiento y apoyo del Estado Colombiano el ente a su vez, debe propender por que la legislación y las políticas de gobierno estén acompañadas de la concertación con las comunidad para evitar decisiones ajenas a las necesidades sociales.

Lo anterior resulta determinante, ya que los jueces de paz y los conciliadores en equidad constituyen herramientas de referencia que la comunidad posee para el reconocimiento de los grupos sociales según sus costumbres y realidades, vistos no solo en grupos de condiciones socio-económicas difíciles, sino de cualquier sociedad que se vea afectada por el deterioro de su tejido social, originado en el conflicto.

Dos trabajos realizados en diferentes épocas ponen de relieve la importancia de ampliar los mecanismos de justicia para generalizar un cambio cultural que incluya nuevas maneras de resolver conflictos societales y proveer un canal de reconocimiento formal para las soluciones encontradas por las partes'.

1 Conciliacion extrajudicial en derecho, Línea de base, Colección Justicia y Seguridad, Departamento Nacional de Planeación, BID, Pontificia universidad Javeriana Bogota, 2004 
El texto "Conciliación extrajudicial en derecho", línea de base del Departamento Nacional de Planeación, (2004), recoge un acucioso estudio bibliográfico de la conciliación extrajudicial en los últimos diez años en nuestro país. Para el presente estudio, hemos retomado varias de las bibliografías analizadas por los investigadores de la Universidad Javeriana. De estas bibliografías, algunas son parte de las que a continuación presentamos.

El estudio realizado por el Centro Interdisciplinario de Estudios Regionales (CIDER) de la Universidad de los Andes (1997), Conflictividad social y el acceso a la justicia, encontró que la relación entre conflictividad social en Bogotá y acceso a la justicia es bajo. El estudio concluye que los altos niveles de conflicto son resueltos de manera violenta, dada la poca socialización colectiva, la cantidad de inmigrantes con poco arraigo a la ciudad y a sus normas, así como la falta de posibilidades de empleo y educación.

La población bogotana tiende a sobrerreaccionar frente a las tensiones y conflictos y hay bajos niveles de socialización de los comportamientos colectivos. Dado que la oferta institucional de mecanismos alternativos de resolución de conflictos que existe hoy en Bogotá es insuficiente para atender la demanda potencial de usuarios de este tipo de servicios, se recomienda al Estado poner en práctica los MASC de manera más contundente, con políticas más eficaces orientadas a la difusión de mecanismos alternos que puedan adelantarse entre pares y fortalecer la oferta institucional existente de justicia administrada con instrumentos de mediación y conciliación.

La investigación realizada por la Sociedad CoIombiana de Pedagogía, Socolpe, denominada “Evaluación y sistematización de las experiencias de conciliación en equidad en 17 municipios de Ios departamentos de Antioquia, Santander y
Cundinamarca" (2001), revela la importancia de la contribución de dichas experiencias a los procesos de paz locales, a la reconstrucción del tejido social y como mecanismo que aporta a la convivencia ciudadana, a la democratización de las relaciones sociales y a la solución de los conflictos con base en valores como la cooperación, la solidaridad, el reconocimiento y el respeto por el otro.

En el campo de la reflexión teórica se encuentra el investigador Jaime Giraldo Ángel (1999), quien enfatiza en su trabajo "Los mecanismos alternativos de solución de conflictos como camino para volver a llenar el derecho de contenido social", que tales mecanismos, como la mediación y la conciliación, se presentan como la posibilidad de introducir en el derecho la problemática social.

La conciliación, al ocuparse del análisis y la solución de los conflictos, abre el campo hasta ahora limitado del ordenamiento jurídico, hacia los espacios sociales y culturales. Se hace necesario transferir los valores, no coercitivamente sino a través de un proceso de culturización. Esta postura se enmarca dentro de la concepción sociológica del derecho, que parte de la base de que éste es un producto cultural al cual el Estado puede también agregarle elementos políticos según los fines que persigue, pero partiendo de reconocerlo como fenómeno social que se desenvuelve dentro del marco de la condicionalidad que la misma realidad le puede ofrecer.

Por su parte, Mario Jaramillo Contreras (1996), en su libro "Justicia por consenso, Introducción a los sistemas alternos de solución de controversias", reconoce que el límite principal para el establecimiento de mecanismos alternos de solución de conflictos tiene que ver con la aplicación del modelo estadounidense al caso colombiano. A pesar de que destaca el papel del Estado y del derecho en la solución de los conflictos, señala sus 
límites en el sentido de no dar las garantías mínimas que exige la convivencia. En este sentido, muestra la necesidad de crear y diseñar mecanismos alternos a los estatales para, estando a tono con las exigencias internacionales, alcanzar la convivencia pacifica.

Aportes a la discusión teórica sobre la solución de conflictos, ofrece el artículo de Germán Palacios (1994) titulado "Resolución alternativa de conflictos: ¿La nueva cara de la política judicial?, resolución alternativa y política judicial". El autor señala que, dado que el contexto político, jurídico y social, enmarcado en el fenómeno general de globalización-glocalización, ha venido ampliando los mecanismos alternativos de resolución de conflictos, importa menos enmarcar la discusión en su carácter de formalidad o informalidad, que en ampliar el marco, hasta ahora exclusivo, de violenciaconflicto. El problema de la distribución de los bienes en una sociedad y la construcción de una cultura que transforme los modos de relación entre las personas y con la naturaleza, se convierte en un aspecto de relevancia mayor.

En este mismo sentido se puede inscribir la discusión que abre el texto de Miguel Sánchez (1995) "Hacia una cultura de la justicia", que aunque no se ocupa específicamente de los mecanismos para la solución de conflictos, su advertencia sobre la necesidad de propiciar una cultura de la justicia, entendida en sentido amplio como valor, aparato y sistema, ofrece pautas para la discusión sobre las relaciones entre justicia y solución de conflictos.

Así mismo, y en un marco más histórico, Hernando Torres (1995) en su artículo "Acceso a la justicia caminos para hacer efectivo el derecho", reconoce los mecanismos extrajudiciales de resolución de conflictos como una de las posibilidades que los ciudadanos tienen para acceder a la justicia. En términos de propuesta, implica pensar programas educativos que, desde el Estado So- cial de Derecho, muevan a los ciudadanos a participar en la construcción de prácticas cotidianas de convivencia.

Otros análisis se centran en destacar el aporte de la conciliación extrajudicial al ordenamiento jurídico. Dentro de ellos, podemos citar los análisis de Juan Carlos Varón (1999) en su artículo "Mecanismos alternativos de solución de conflictos, avances de la conciliación extrajudicial civil y comercial". El autor hace un reconocimiento a la conciliación extrajudicial, destacando su definición, objeto, características y aplicación, como mecanismo de respuesta a la crisis de violencia en Colombia y como aporte al rediseño de la función social de la justicia.

Señala, entre los beneficios del régimen de conciliación, los bajos costos y la producción de una menor tensión en las relaciones entre las partes. Igualmente, y de manera particular, el autor señala las limitaciones que afectan la conciliación de controversias en la esfera civil y comercial así como pone de relieve las carencias en la profesionalización de los conciliadores.

En general, destaca la necesidad de fortalecer los centros de conciliación (inclusión de personal competente y capacitado, creación de nuevos centros de carácter interdisciplinario, expedición de un código de ética del conciliador, campañas de concienciación ciudadana, entre otros) y de canalizar más controversias referentes a la esfera civil y comercial (incumplimiento de contratos, no pago de obligaciones, reclamo de compensación económica por accidentes, liquidación de sociedades, entre otros).

Otro tipo de análisis también muestra el debate vigente acerca del requisito de procedibilidad. Dentro del marco restringido al análisis de la Ley 640 de conciliación y en especial en la conciliación de familia, a partir del año 2002, una de las posturas más críticas se encuentra en el artículo de 
Alejandro Bernal (2001) denominado "Ley 640/ 2001. La conciliación en familia a partir del 2002". Este trabajo, si bien deja de lado el contexto sociocultural, advierte sobre la necesidad de estimular el debate frente a lo que el autor considera los principales efectos de la ley, tales como la imposición de la conciliación, la privatización y el encarecimiento de la justicia.

En esta misma línea, Norma Gálvez y Paola Torrente (2001) destacan en su artículo "Acuerdos y desacuerdos en torno a la conciliación en CoIombia", el trabajo realizado por la Corporación Excelencia en la Justicia en las áreas de gestión, educación y acceso a la justicia en el marco de los servicios formales e informales de administración de justicia. Concluye que la apuesta del gobierno por fortalecer la conciliación mediante la obligatoriedad del procedimiento, implica ampliar y cualificar la oferta y el servicio. Se debe hacer que los centros de conciliación presten de forma gratuita sus servicios. Se requiere, además, propiciar escenarios de concertación para la búsqueda de acuerdos adicionales a favor del acceso ciudadano a la justicia.

Los análisis presentados por Esteban Reyes (2002), investigador de la facultad de derecho de la Universidad de los Andes, en su artículo "La conciliación como requisito de procedibilidad en la jurisdicción civil en Colombia", muestran los beneficios de la conciliación para el sistema judicial en términos de descongestión del aparato judicial y de reducción de los costos de la justicia, al tiempo que señalan la importancia de maximizar el desempeño de los centros de conciliación en aspectos éticosociales, pedagógicos, psicológicos y culturales.

Algunos análisis realizados a solicitud del Ministerio del Interior y de Justicia, han profundizado sobre los procesos de análisis y evaluación de gestión del mecanismo de la conciliación y de las estrategias jurídicas que le han dado viabili- dad en la práctica, tratando de establecer precisiones acerca del comportamiento respecto a los objetivos que les dieron origen y determinar el grado de evolución de la figura, ratificada sistemáticamente a través de los desarrollos normativos. En realidad, de acuerdo con la revisión en curso, los estudios de desempeño de la figura de la conciliación y de las leyes que la conciernen, son escasos.

En el proceso de la revisión bibliográfica se han encontrado otros estudios sobre la Conciliación Extrajudicial en Derecho, que describen aspectos como los orígenes del mecanismo desde el punto de vista legal y su relación con los métodos alternativos de resolución de conflictos; se efectúan comparaciones normativas o se señalan los avances de nuevas leyes, resoluciones o decretos. Es un enfoque evaluativo-analítico que sugiere la importancia de crear y adoptar instrumentos de seguimiento para la práctica de la conciliación basado en indicadores de gestión.

Esta revisión bibliográfica ha mostrado el énfasis de los trabajos realizados para el Ministerio en el tema de las políticas adoptadas para el fortalecimiento del sector justicia en el tema de la descongestión de los despachos judiciales, sin dejar de considerar la importancia atribuida al acceso a la justicia. Entre estas políticas se incluye la promoción de los mecanismos alternativos de resolución de conflictos como medios idóneos para contribuir a generar eficiencia en el aparato judicial y a la vez producir cambios culturales respecto a la forma de enfrentar conflictos de diversa naturaleza.

Los estudios muestran que el interés primero de estas reformas políticas ha pretendido desjudicializar e informalizar el trámite de los conflictos con el fin de descongestionar el sistema judicial (Tolosa, 1998). El estudio contratado por el BID reveló no sólo una enorme congestión en los despachos judiciales sino "la nega- 
ción del acceso a la justicia como la más nefasta consecuencia de la disfuncionalidad del aparato de Estado" (s. p.).

Los resultados de la consultoría realizada por Tolosa indican varias recomendaciones en diferentes aspectos:

Lograr un manejo más integral de los conflictos y por tanto una mejor calidad en la prestación de los servicios y la transformación constructiva de las controversias. La interdisciplinariedad es una apuesta que debe ser promovida para sacar a los MASC del exclusivo ámbito jurídico irradiándolos a otras esferas del conocimiento, particularmente desde las ciencias humanas.

Otra recomendación destaca la función evaluativa de los entes estatales en cuanto a la gestión e impacto de la Conciliación Extrajudicial en Derecho para lo cual el investigador mencionó:

Es urgente el diseño de un formato que permita la periódica y homogénea recepción de la información requerida por la División de parte de cada uno de los centros en relación con la prestación del servicio. Este formato debe permitir establecer semestralmente, con cifras estadísticas claras, el desarrollo e impacto de la utilización de la figura en cuanto a: acceso al servicio, deserción de usuarios, número de acuerdos logrados (total y parcial) y materia "(Tolosa, 1998).

La investigación realizada por el Centro de Investigaciones para el Desarrollo (CID) de la Universidad Nacional de Colombia (1999) en un trabajo realizado para el Ministerio de Justicia y del Derecho, "Diseño de la metodología de evaluación técnica y financiera para la creación de centros de conciliación y arbitraje y el establecimiento de tarifas oficiales de los servicios ofrecidos", muestra la necesidad de establecer una metodología de factibilidad para la presentación y evaluación de solicitudes de creación de Centros de Conciliación y arbitraje ante el Ministerio de Justicia y del Derecho y la necesidad de fijar un marco tarifario para los servicios ofrecidos por estos centros. El estudio provee herramientas y parámetros para la valoración de las solicitudes de creación de dichos centros y un marco de tarifas que continúan vigentes en la actualidad.

El texto de Guillermo Chaín Lízcano (1995) “El concepto de justicia en la realidad colombiana", discute el concepto de justicia en el contexto colombiano desde una tendencia a identificar la justicia con el aparato administrativo encargado de ejecutarla, justicia como virtud y como instrumento para el ejercicio de la autoridad. Concluye que aunque la conciliación extrajudicial en derecho tuvo su origen en la necesidad social de administrar justicia de manera eficaz, legítima y eficiente, aún quedan dudas sobre su eficacia, eficiencia, suficiencia y legitimidad para satisfacer las demandas sociales en la administración de la justicia. Es necesario brindar recursos pedagógicos que conduzcan al pueblo a acunar el hábito de lo justo. Para ello se debe indagar sobre los hechos del diario acontecer que puedan considerarse justos o injustos.

Informes evaluativos sobre las leyes que conciernen la introducción de la conciliación muestran otro plano del estado del arte en esta materia:

El Instituto de Estudios Interdisciplinarios de la Universidad Externado de Colombia (1994) realizó una "Evaluación del rendimiento de la Ley de conciliación". Dicha evaluación establece el censo del número de personas que está haciendo conciliaciones a partir de la implementación de la Ley 23 de 1991, en sus aspectos de conciliación obligatoria al interior del proceso judicial y en su forma extrajudicial en centros de conciliación privados y autoridades con competencia para conciliar. Sin embargo el balance de resultados no es concluyente.

El informe evaluativo de la Ley 446 de 1998, elaborado por el Ministerio de Justicia y presentado al Congreso de la República, tomando como po- 
blación objetivo del estudio las facultades de derecho y los abogados litigantes, señala que, mientras las primeras consideran que la aplicación de la figura de la conciliación ha sido alta y su divulgación y aplicación deber ser fortalecida, los segundos estiman que su aplicación al ejercicio profesional ha sido regular o baja y es necesario clarificar las competencias. En general, se reconoce que luego de la expedición e implementación de dicha ley, ha habido una mejoría en la administración de justicia.

En términos generales, el informe en referencia enfatiza que la aplicación y fortalecimiento de los mecanismos alternativos de resolución de conflictos se considera:

La justicia del futuro, puesto que existe la necesidad de cambiar los esquemas tradicionales de la justicia formal. Se trata de una nueva forma de justicia que, además de resolver; previene los conflictos y evita la congestión del aparato judicial dada su rapidez, eficiencia y economía (Bautista Pérez y otros, Ministerio de Justicia, 2000: 73).

\section{CONCLUSIONES PARCIALES}

Frente a la investigación anterior se hace evidente el gran avance que han tenido los MASC desde 1991 hasta la fecha. El Estado colombiano ha tratado de dar buena cuenta y aplicación a lo establecido por el constituyente y por el legislador en la normatividad descrita, poniendo, de esta manera, en la mira dos elementos esenciales como un componente legislativo y regulador que cubre con gran amplitud y da cabida a nuevos elementos y herramientas para el acceso de las comunidades a la justicia de manera uniforme e igualitaria; un segundo componente "campo de aplicación", daría lugar a más cobertura y eficacia de las "nuevas" propuestas en justicia comunitaria, de paz y alternativa y/o en equidad, de acuerdo al modo en que se utilice el termino, pues resulta discutible el empleo de estas palabras frente a su definición (cuestión que no es tema de esta investigación, pero frente a la cual corresponde hacer referencia por el constante desacierto en el empleo de estos vocablos para hacer alusión a los diferentes tipos de justicia referenciados en el material, objeto de investigación.

Consideramos que a pesar de la preocupación del Estado por dar aplicación a estos mecanismos para no congestionar mas los despachos judiciales, le ha faltado dar información y divulgación de las figuras de los mecanismo alternativos de resolución de conflictos (MASC), de la justicia alternativa y en equidad, además de ofrecer capacitación suficiente a los miembros de la sociedad para que la aplicación de estos mecanismos se ofrezcan bajo los lineamientos sociales, legales e institucionales correspondientes a las necesidades de cada contexto.

La falta de divulgación de la justicia de paz, la conciliación en equidad, las carencias de tipo económico, material y humano, dificultan su funcionamiento, utilidad, servicio, eficiencia y eficacia, tanto en las comunidades urbanas como en las rurales, impidiendo el acceso a estos modelos de justicia por parte de los diferentes sectores sociales.

\section{BIBLIOGRAFIA}

Abella, M. C. \& Vargas, A. (2000). Justicia de paz y solución de conflictos cotidianos en Neiva, Ibagué y Florencia. Universidad sur colombiana de Neiva.

Álvarez M., Corzo, L. \& Figueroa, D. (2001). Jueces de paz una figura de la justicia comunitaria análisis. Procuraduría general de la nación. 
Bautista, A. M. (2000). Evaluación sobre la aplicación de la Ley 446 de 1998. Informe al Congreso de la Republica. Bogotá: Ministerio de Justicia.

Bernal, A. (2001). Ley 640 2001. La Conciliación en familia a partir de 2002. En Revista del Colegio de jueces y fiscales de Antioquia 20. Medellín: Universidad Nacional de Colombia.

Calvo, G. (1995). Estado del arte de la familia en Colombia 1980-1994. Ministerio de salud. Instituto Colombiano de bienestar familiar.

Corvalán G. (1985). El bilingüismo en América Latina. Ind. En La educación 30, 98 .

Galvez, N. \& Torrente, P. (2001). Acuerdos y desacuerdos entorno a la conciliación en Colombia. En Revista Justicia y Desarrollo 17. pp. 4959. Bogotá: Corporación Excelencia en la Justicia.

Giraldo, A. (1999). Los mecanismos alternativos de solución de conflictos como camino para volver a llenar el derecho de contenido social, primera parte: Mecanismos de resolución de conflictos. En Contexto y Conflicto. Bogotá: Tercer Mundo.

Hoyos, C. (2000). Un modelo para investigación documental. Señal editora.

Jaramillo, M. (1996). Justicia por consenso. Introducción a los sistemas alternos de solución de Controversias. Bogotá: Institución Universitaria Sergio Arboleda.

Lee Van Cott, D. (2000). A political analysis of legal pluralism in Bolivia and Colombia. En
Journal of Latin American studie.

Liscano, Ch. (1995). El concepto de justicia en la realidad colombiana. En Revista Pensamiento Jurídico 4. Bogotá: Universidad Nacional de Colombia. Facultad de Derecho, Ciencias Políticas y Sociales.

Rengifo, A. (1998). Mecanismos alternativos de solución de conflictos. Ministerio de Justicia.

Reyes, E. (2002). La Conciliación como requisito de procedibilidad en la Jurisdicción Civil en Colombia. En Revista Derecho Privado No. 28. Bogotá.

Rinaudo, U. (1997). Conflictividad social y acceso a la justicia (Informe Final). Centro Interdisciplinario de estudios Regionales CIDER. Bogotá: Universidad de los Andes.

Rodríguez, P. (2001). Las decisiones administrativas y la conciliación como mecanismo alternativo de resolución de conflictos. Colciencias.

Ross, M. H. (1995). La Cultura del Conflicto. BarceIona: Paidos.

Sánchez, M. (1995). Hacia una cultura de la justicia. En Revista Pensamiento Jurídico 4. Bogotá: Universidad Nacional de Colombia.

Sociedad Colombiana de Pedagogía. (2001). Evaluación y sistematización de las experiencias de conciliación en equidad en 17 municipios de los Departamentos de Antioquia, Santander y Cundinamarca. Bogotá: Autor.

Stapper, A. F. \& Solórzano M. (2004). Mediación Comunitaria. Tesis de grado. Universidad Santo Tomás, Bogotá, Colombia. 
Tolosa, W. (1998). Programa de conciliación institucional evaluación primera fase.. Bogotá: Ministerio de Justicia y del Derecho.

Torres, H. (1995). Acceso a la justicia, caminos para hacer efectivo el Derecho. En Revista Pensamiento Jurídico 4. Bogotá: Universidad Nacional de Colombia.
Varón, J. C. (1999). Avances de la conciliación extrajudicial, civil y comercial. En Revista de la Corporación Excelencia de la Justicia. Bogotá.

(2002). Mecanismos alternativos de solución de conflictos, avances de la conciliación extrajudicial civil y comercial. En Revista de Derecho Privado 28. Bogotá. 